Pesq. Vet. Bras. 36(9):901-904, setembro 2016 DOI: $10.1590 / \mathrm{S} 0100-736 \mathrm{X} 2016000900017$

\title{
Descrição anatômica do plexo braquial de Callithrix jacchus e Callithrix penicillata ${ }^{1}$
}

\author{
Paulo R.S. Santos ${ }^{2 *}$, Matheus Henrique R. Silva ${ }^{3}$, Amanda R. Rodrigues ${ }^{2}$ \\ e Antônio C. Assis Neto ${ }^{2}$
}

\begin{abstract}
Santos P.R.S., Silva M.H.R., Rodrigues A.R. \& Assis Neto A.C. 2016. [Anatomic description of brachial plexus of Callithrix jacchus and Callithrix penicillata.] Descrição anatômica do plexo braquial de Callithrix jacchus e Callithrix penicillata. Pesquisa Veterinária Brasileira 36(9):901-904. Programa de Pós-Graduação em Anatomia dos Animais Domésticos e Silvestres, Departamento de Cirurgia, Setor Anatomia, Faculdade de Medicina Veterinária e Zootecnia (FMVZ), Universidade de São Paulo (USP), Av. Prof. Dr. Orlando Marques de Paiva 87, São Paulo, SP 05508-270, Brazil. E-mail: pauloramos@usp.br

Callithrix jacchus and Callithrix penicillata are small primates used as anatomic model, not only for convenience in handling as ease in regard to breeding and reproductive rate. The aim of this study was to describe the components of the brachial plexus in Callithrix jacchus and C. penicillata. Three specimens about 8 years old and weighing $240 \mathrm{~g}$ were fixed in $10 \%$ formaldehyde and subsequently dissected and photodocumented. The brachial plexus of Callithrix jacchus and C. penicillata originates from the spinal nerves C5 to T1 in continuation of the cranial, medium and flow trunk. The composition of the brachial plexus of these animals is similar to the one of other primates ands other mammals.
\end{abstract}

INDEX TERMS: Brachial plexus, Callithrix jacchus, Callithrix penicillata, white tuft marmoset, black tuft marmoset, nervous system, peripheral nerves.

RESUMO-- Callithrix jacchus e Callithrix penicillata são primatas de pequeno porte cuja utilização como modelo anatômico tem se mostrado cada vez mais frequente, não somente pela praticidade no manuseio como facilidade no trato em criatório e sua taxa de reprodução. Este estudo teve como objetivo descrever os componentes dos plexos braquial em Callithrix jacchus e penicillata. Para tanto, três espécimes com aproximadamente 8 anos e $240 \mathrm{~g}$ foram fixados em solução de formaldeído a 10\%, e posteriormente dissecados e fotodocumentados. 0 plexo braquial do Callithrix jacchus e penicillata originou-se dos nervos espinhais C5 a T1 constituindo os troncos cranial, médio e caudal. A composição do plexo braquial destes animais se assemelha ao de outros primatas, bem como a outros mamíferos.

\footnotetext{
${ }^{1}$ Recebido em 26 de agosto de 2015.

Aceito para publicação em 12 de maio de 2016.

Programa de Pós-Graduação em Anatomia dos Animais Domésticos e Silvestres, Departamento de Cirurgia, Setor Anatomia, Faculdade de Medicina Veterinária e Zootecnia (FMVZ), Universidade de São Paulo (USP), Av. Prof. Dr. Orlando Marques de Paiva 87, São Paulo, SP 05508-270, Brasil. *Autor para correspondência: pauloramos@usp.br

${ }^{3}$ Centro Universitário Adventista de São Paulo (Unasp), Estrada de Itapecerica 5859, São Paulo, SP 05858-001, Brasil.
}

TERMO DE INDEXAÇÃO: Plexo braquial, Callithrix jacchus, Callithrix penicillata, sagui de tufo branco, sagui de tufo preto, sistema nervoso, nervos periféricos.

\section{INTRODUÇÃO}

Os saguis de tufo branco e de tufo preto (Callithrix jaccus e Callithrix penicillata) são primatas diurnos cujas características sociais e reprodutivas, associadas à facilidade no trato e seu pequeno porte, os tornam modelos apropriados para pesquisas de comportamento, reprodução, infectologia e neurocientificas (Mansfield 2003).

Endêmicos do Brasil, os saguis de tufos brancos e saguis de tufos pretos não estão entre os animais em risco de extinção. Pelo contrário, a ação antrópica sobre os animais e seu habitat (principalmente mata atlântica e cerrado) tem gerado a disseminação destes primatas para regiões onde estes passam a ser invasores, colocando em risco outras espécies (Ruiz-Miranda et al. 2006, Morais Jr 2010).

Diferentemente de outros primatas, saguis possuem uma dieta abrangente que inclui insetos, moluscos, frutos, exsudados, pequenos vertebrados, sementes, ovos de aves e néctar. Saguis vivem em famílias, as fêmeas possuem gestação de 5 meses e, em sua maioria, resulta na geração de gêmeos (Abbott et al. 2003, Reis et al. 2006). 
Sua dieta, taxa de reprodução e seu porte pequeno favorecem seu uso em pesquisas biomédicas, já sendo um modelo comum na Europa e EUA (Mansfield 2003), bem como seu uso no desenvolvimento de técnicas mais eficientes de bloqueios anestésicos locais e técnicas cirúrgicas que objetivam a reparação de danos causados em nervos periféricos (Glasby et al. 1992, Liu et al. 1998).

Devido à sua localização, o plexo braquial é especialmente suscetível a lesões, uma vez que, além de sofrer tração por parte das estruturas que inerva, não possui estruturas ósseas que forneçam proteção (Flores 2006). 0 trabalho teve como objetivo descrever o plexo braquial de Callithrix jacchus e penicilatta em sua origem, composição, e nervos derivados, podendo assim servir de conhecimento anatômico comparativo e em procedimentos anestésicos.

\section{MATERIAL E MÉTODOS}

Foram utilizados dois saguis machos, sendo um exemplar Callithrix penicillata (tufos preto, IBAMA- 963000109258) e dois exemplares de Callithrix jacchus (tufo branco, IBAMA-96300072274), de 190-280g, com idade mínima de 8 anos de idade, moreram por causas naturais, foram cedidos pelo Criadouro Comercial AJBSOARES (IBAMA no2029), situado no município de Atibaia, São Paulo, para o Setor de Anatomia da Faculdade de Medicina Veterinária e Zootecnia, Universidade de São Paulo.

Os animais foram fixados em formol a $10 \%$ e posteriormente dissecados. A pele da região torácica e dos braços foi rebatida; a porção ventral da coluna cervical e torácica foi aberta cuidadosamente, e em seguida procedeu-se com a remoção do músculo escaleno expondo as raízes do plexo braquial seguindo o trajeto dos troncos e nervos originários para a identificação da origem e dos nervos que compõem o plexo.

A visualização das estruturas foi facilitada através da utilização de duas técnicas. Ao Callithrix jacchus foi adicionado uma solução de ácido acético/álcool absoluto 30\%. Os nervos ficaram em contato com a solução por $15 \mathrm{~h}$ consecutivas e, durante as dissecações a solução foi aplicada aos nervos expostos objetivando o clareamento das estruturas (Ribeiro 2002). E ao Callithrix penicillata, foi adicionada água oxigenada 20 volumes por $12 \mathrm{~h}$ e, tal qual com o ácido, a solução foi acrescida à medida que novas estruturas fo- ram expostas. A terminologia adotada foi baseada no International Committee on Veterinary Gross Anatomical Nomenclature (2012).

\section{RESULTADOS E DISCUSSÃO}

As observações feitas em Callithrix jacchus e em C. penicillata revelaram que possuem sete vertebras cervicais, com emergência do primeiro par de nervos espinhais entre o occipital e o atlas. 0 plexo braquial é constituído por fibras neurais provenientes do quinto, sexto, sétimo e oitavo cervicais, e do primeiro torácico (Fig.1A), e que correspondem às vértebras cervicais $5^{\circ}, 6^{\circ}, 7^{\circ} \circ, 8^{\circ}$ e à $1^{\circ}$ o torácica. Assim podemos mostrar que a distribuição dos nervos do plexo braquial tem um limite superior em C5 e um limite inferior em T1. Com isso, os nervos cervicais C5 e C6 formam o tronco cranial, o C7 forma o tronco médio, e o C8 a T1 formam o tronco caudal (Fig.1A).

A origem do plexo braquial de Callithrix apresentaram semelhanças aos outros primatas estudados. Em espécies de macaca, foi observado origens da quinta medula cervical ao primeiro medular torácico, podendo também iniciar a partir da quarta medular cervical (Brooks 1883). Babuínos (Booth et al. 1997), orangotango (Kawashima et al. 2007), macaco-de-cheiro (Mizuno 1969) e macaco barrigudo (Cruz \& Adami 2010) são semelhantes ao Callithrix, com origem entre C5 a T1. Hill (1972), observou que Lagothrix sp. difere dos outros primatas, onde apresenta limites do plexo entre C4 e T2. A formação dos troncos cranial, médio e caudal se assemelha aos encontrados em outros primatas, como o babuíno (Booth et al. 1997), macaca (Sugiyama 1965); gorila (Koizumi \& Sakai 1995), macaco-prego (Sugiyama 1965, Ribeiro 2002), entre outros.

Os troncos cranial, médio e caudal, formados pelos nervos cervicais originaram quase todos os nervos do plexo braquial em Callithrix jacchus e C. penicillata (Quadro 1). 0 tronco cranial deu origem aos nervos subescapular (NSB), nervo supraescapular (NSP), nervo mediano (NM), nervo radial (NR), nervo musculocutâneo (NMC), e o nervo axilar (NA) (Fig.1B). 0 tronco médio originou os nervos peitorais
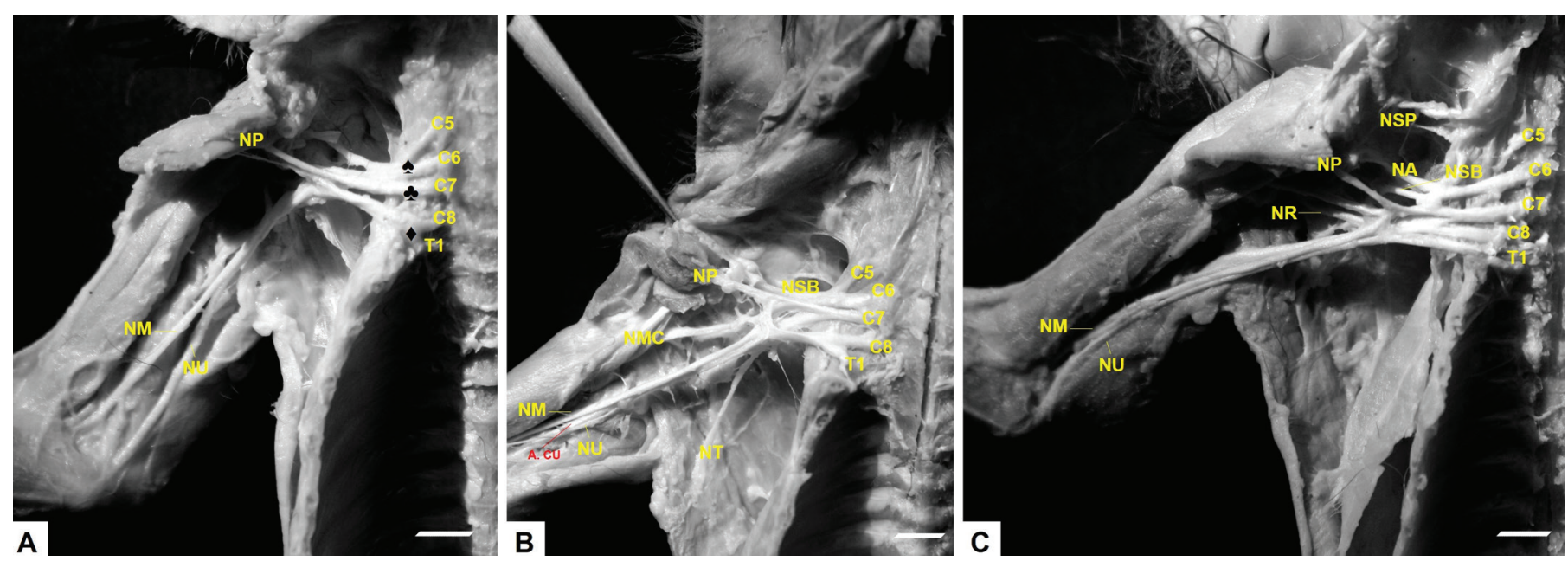

Fig.1. Vista ventral do antímero direito de Callithrix. Notam-se os troncos: cranial (\$), médio ( $\bullet$ ), caudal ( ). Observam-se: artéria colateral ulnar (A. CU), nervo peitoral (NP), nervo subescapular (NSB), nervo supraescapular (NSP), nervo mediano (NM), nervo ulnar (NU), nervo radial (NR), nervo musculocutâneo (NMC), nervo torácico lateral (NT), e nervo axilar (NA). (A,B) Callithrix jacchus. (C) Callithrix penicillata. 
Quadro 1. Plexo braquial de Callithrix penicillata e . jacchus

\begin{tabular}{lll}
\hline \multicolumn{1}{c}{ Nervo } & \multicolumn{1}{c}{ Origem } & \multicolumn{1}{c}{ Inserção } \\
\hline Peitoral (NP) & Tronco médio & M. peitorais \\
Subescapular (NSB) & Tronco cranial/médio & M. subescapular \\
Supraescapular (NSP) & Tronco cranial & M. supraespinhoso/infraespinhoso \\
Mediano (NM) & Troncos: cranial/médio/caudal & M. braquial/bíceps braquial/tríceps braquial \\
Ulnar (NU) & Tronco caudal & M. tríceps braquial \\
Radial (NR) & Troncos: cranial/médio/caudal & M. tríceps braquial \\
Musculocutâneo (NMC) & Tronco cranial & M. coracobraquial \\
Torácico Lateral (NT) & Tronco cranial & M. grande do dorso/peitoral profundo \\
Axilar (NA) & Tronco cranial/médio & M. redondo maior/redondo menor
\end{tabular}

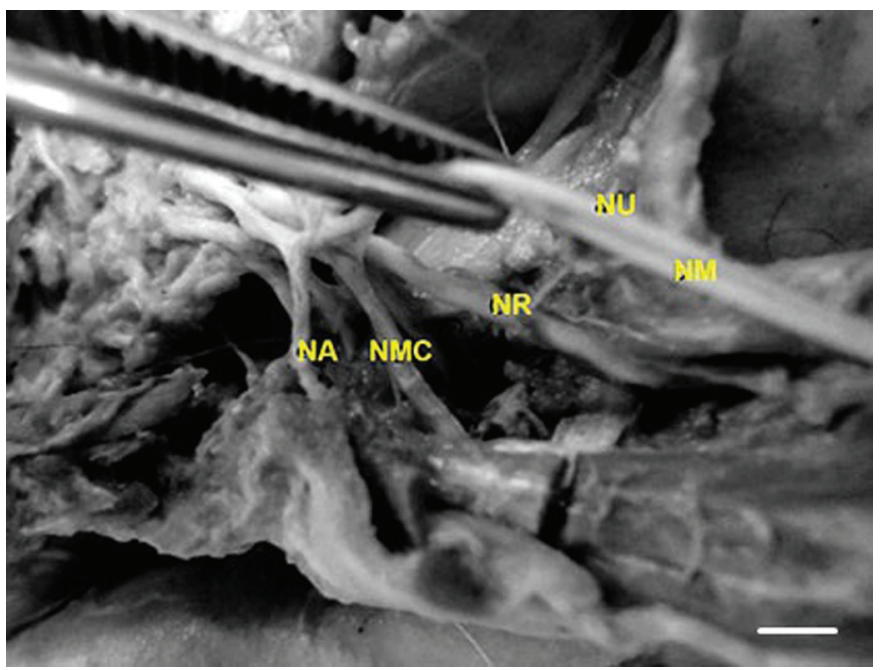

Fig.2. Vista ventro-medial do antímero direito de Callithrix jacchus. Nervo axilar (NA), nervo musculocutâneo (NMC), nervo radial (NR), nervo mediano (NM), e nervo ulnar (NU).

(NP), nervo subescapular (NSB), nervo mediano (NM), nervo radial (NR), e nervo axilar (NR) (Fig.2). 0 tronco caudal originou os nervos mediano (NM), nervo ulnar (NU), nervo radial (NR), e o nervo torácico lateral (NT) (Fig.1C).

$O$ nervo subescapular (NSB) em Callithrix jacchus e $C$. penicillata, macaco-barrigudo e em babuínos, apresentaram origem a partir do tronco cranial (Booth et al. 1997, Cruz \& Adami 2010). Já o nervo supraescapular (NSP) apresentou a mesma origem do chimpanzé e babuíno (Hepburn 1892, Booth et al. 1997). Os troncos cranial, médio e caudal, contribuíram para a formação do nervo mediano (NM) em Callithrix jacchus e C. penicillata, fato também observado em babuínos e no macaco-barrigudo (Booth et al. 1997). A origem do nervo radial (NR) em Callithrix jacchus e C. penicillata está relacionado com a origem dos demais nervos do plexo braquial, semelhante ao visto em babuínos (Booth et al. 1997), outros primatas como o macaco-prego apresentam uma origem a partir de C6, tronco médio e C8 (Ribeiro 2002). Em Callithrix jacchus e C. penicillata, babuíno e macaco-barrigudo, o nervo musculocutâneo originou-se a partir do tronco cranial.

Assim como em babuínos (Booth et al. 1997), o nervo peitoral (NP) de Callithrix jacchus e C. penicillata se originou do tronco médio, diferindo de outros primatas, como o chimpanzé, o orangotango e gorila, que apresentam origem nos troncos cranial e médio (Champneys 1975, Hepburn 1982). O nervo torácico lateral (NT) de Callithrix jacchus e C. penicillatae, como também de outros mamíferos, são originados do tronco caudal (C8 e T1) (Scavone et al. 2008, Chagas et al. 2014).

O nervo ulnar (NU) em Callithrix jacchus e C. penicillata originou-se a partir de um tronco comum com o nervo mediano (NM), recebendo fibras vindas de C8, T1. Em babuínos (Papio ursinus) o NU originou-se de um tronco comum com o NM e o NCMA recebendo fibras de C8, T1 (tronco caudal), a mesma disposição foi encontrada em babuínos (Booth et al. 1997). Foi observado que o nervo ulnar (NU) de Callithrix penicillata não se separou do nervo mediano (NM) até aproximarem a região da articulação úmero radio ulnar, fato que também foi observado por Mizuno (1969) em macaco-prego-de-cara-branca.

\section{CONCLUSÕES}

O plexo braquial de Callithrix jacchus e C. penicillata origina-se dos nervos espinhais $\mathrm{C} 5$ a T1 constituindo os troncos cranial, médio e caudal.

Sugere-se que o plexo braquial destes animais se assemelha ao dos outros primatas, bem como a outros mamíferos.

\section{REFERÊNCIAS}

Abbott D.H., Barnett D.K., Colman R.J., Yamamoto M.E. \& Schultz-Darken N.J. 2003. Aspects of common marmoset basic biology and life history important for biomedical research. Comp. Med. 53(4):339-350.

Booth K.K., Baloyi F.M. \& Lukhele O.M. 1997. The brachial plexus in the Chacma baboon (Papio ursinus). J. Med. Primatol. 26:196-203.

Brooks W.T. 1883. The brachial plexus of the Macaque monkey and its analogy with that of man. J. Anat. Physiol. 17(3):329-332.

Chagas K.L.S., Fé L.C.M., Pereira L.C., Branco E. \& Lima A.R. 2014. Descrição morfológica do plexo braquial de jaguatirica (Leopardus pardalis). Biotemas 27(2):171-176.

Champneys F. 1975. On the muscles and nerve of a Chimpanzee (Troglodytes niger) and Cynocephalus anubis. J. Anat. Physiol. 6(1):176-211.

Cruz G.A.M. \& Adami M. 2010. Anatomia do plexo braquial de macaco-barrigudo (Lagothrix lagothricha). Pesq.Vet. Bras. 30(10):881-886.

Flores L.P. 2006. Estudo epidemiológico das lesões traumáticas de plexo braquial em adultos. Arq. Neuropsiquiatr. 64(1): 88-94.

Glasby M.A., Carrick M.J. \& Hems T.E.J. 1992. Freeze-thawed skeletal muscle autografts used for brachial plexus repair in the non-human primate. J. Hand Surgery 17B:526-535

Hepburn D. 1892. The comparative anatomy of the muscles and nerves of the superior and inferior extremities of the anthropoid apes. Part I. J. Anat. Physiol. 26(2):149-186.

Hill W.C.O. 1972. Primates: Comparative anatomy and taxonomy. V. Cebidae: Part B. Edinburgh University Press, Edinburgh. 537p.

International Committee on Veterinary Gross Anatomical Nomenclature 2012. Nomina Anatomica Veterinaria. 5th ed. World Association of Vet- 
erinary Anatomists, Hannover (Germany), Columbia, MO (USA), Ghent (Belgium), Sapporo (Japan).

Kawashima T., Yoshitomi S. \& Sasaki H. 2007. Nerve fibre tracing of branches to the coracobrachialis muscle in a Bornean orangutan (Pongo pygmaeus pygmaeus). Anat. Histol. Embryol. 36(1):19-23.

Liu S., Bodjarian N., Langlois O., Bonnard A.S., Boisset N., Peulvé P., Saïd G. \& Tadié M. 1998. Axonal regrowth through a collagen guidance channel bridging spinal cord to the avulsed $\mathrm{C} 6$ roots: functional recovery in primates with brachial plexus injury. J. Neurosci. Res. 51:723-734.

Mansfield K. 2003. Marmoset models commonly used in biomedical research. Comp. Med. 53(4):383-392.

Mizuno N. 1969. The brachial plexus of a Capuchin Monkey (Cebus capucinus). Primates 10:37-40.

Morais Jr M.M. 2010. Os saguis (Callithrix spp. Erxleben, 1777) exóticos invasores na bacia do rio São João, Rio de Janeiro: biologia populacional e padrão de distribuição em uma paisagem fragmentada. Universidade Estadual do Norte Fluminense Darcy Ribeiro, Campos dos Goytacazes, RJ.
Reis N.R., Peracchi A.L., Pedro W.A. \& Lima I.P. 2006. Mamíferos do Brasil. FURB, Londrina.

Ribeiro A.R. 2002. Estudo anatômico do plexo braquial do macaco Cebus apella: origem, composição e nervos resultantes. Dissertação de Mestrado em Anatomia dos Animais Domésticos, Faculdade de Medicina Veterinária e Zootecnia, USP, São Paulo. 146p.

Ruiz-Miranda C.R., Affonso A., Morais M.M., Verona C., Martins A. \& Beck B.B. 2006. Behavioral and ecological interactions between reintroduced golden lion tamarins (Leontopithecus rosalia Linnaeus, 1766) and introduced marmosets (Callithrix spp. Linnaeus, 1758) in Brazil's Atlantic coast forest fragments. Braz. Archs Biol. Technol. 49:99-109.

Scavone A.R.F., Machado M.R.F., Guimarães G.C., Oliveira F.S. \& Gerbasi S.H.B. 2008. Análise da origem e distribuição dos nervos periféricos do plexo braquial da paca (Agouti paca Linnaeus, 1766). Ciênc. Anim. Bras. 9(4):1046-1055.

Sugiyama T. 1965. On the plexus brachialis of Macacus cyclopsis. Acta Med. Nagasaki 9(3):65-68. 